\title{
Connecting the Dots: Stages of Implementation, Wraparound Fidelity and Youth Outcomes
}

\author{
Vicki Sprague Effland, Betty A. Walton, \& Janet S. McIntyre
}

\begin{abstract}
Several necessary system and organizational support conditions for wraparound have been identified (Walker et al. 2003). Yet, the relationship between these necessary system level conditions and wraparound fidelity has only recently begun to be examined. Similarly, few studies have included a measure of wraparound fidelity when examining the relationship between wraparound implementation and youth outcomes. The statewide implementation of a wraparound demonstration grant offers the opportunity to explore these relationships and to identify factors that predict improvement in functioning for youth receiving wraparound. Findings suggest that significant relationships exist between (1) the stage of development of necessary support conditions for wraparound and wraparound fidelity and (2) wraparound fidelity and improvement in youth outcomes. Specific elements of wraparound (i.e., outcomes based and community based) and baseline needs and strengths (e.g., high levels of anxiety and conduct issues, poor functioning at home and in school, judgment, and risks) were found to predict a reduction in youth needs. Other unexpected relationships between youth outcomes and the cultural competence element of wraparound and being multi-racial were also discovered. These findings reinforce the importance of supporting high fidelity wraparound for youth and their families in a recovery focused behavioral health system.
\end{abstract}

\section{Introduction}

Wraparound is a family-driven, youth guided, team-based process for planning and implementing services and supports (Miles et al. 2006). The National Wraparound Initiative (NWI) has identified ten elements of wraparound (i.e., family voice and choice, team based, natural supports, collaborative, community based, culturally competent, individualized, strengths based, persistence and outcomes based) and four phases through which teams consisting of the identified youth, his/her parents or caregivers, family members, community members, mental health professionals, and others are expected to move as they develop and implement a single plan of care. The plan of care includes the services and supports necessary to build on the strengths of the youth and his/her family and addresses the complex needs of the youth involved in the wraparound process.

Emerging evidence supports the effectiveness of wraparound for youth who have needs in multiple life domains (e.g., home, school and community). Nine controlled studies of wraparound (see Bruns and Suter 2010; Suter and Bruns 2009) found improved outcomes for youth in wraparound compared to similar youth in other programs, with effect sizes similar to those found in studies of other evidence based interventions implemented in real world practice (Suter and Bruns 2009). However, only one of the nine studies considered the relationship between wraparound fidelity and outcomes (Bruns et al. 2006). Research on other evidencebased practices has repeatedly found that fidelity to the practice model is vital to outcomes (e.g., Henggeler et al. 1997; McGrew et al. 1994; Walton 2006). Additional research on wraparound that includes a measure of fidelity as well as further research on the factors that predict successful outcomes for youth involved in the wraparound process are needed (Bruns et al. 2010; Cox et al. 2010).

Successful implementation of any practice 
model depends on several key community and organizational factors, such as organizational support, staff selection, training, coaching, and systemlevel partnerships (Glisson and Schoenwald 2005; Lehman et al. 2002; Metz et al. 2007). A few authors have discussed similar factors that are associated with effective wraparound implementation (e.g., Bertram et al. 2010; Walker 2008; Walker et al. 2003). For example, Walker et al. (2003) identified several necessary conditions to support the implementation of high-quality individualized planning, such as wraparound, at the system, organization and team levels. Studies examining the relationship between these necessary conditions and wraparound fidelity and/or outcomes are only just emerging (Bruns et al. 2006; Stephens et al. 2004; Walker and Sanders 2010). Understanding these relationships is essential to reforming child-serving systems to effectively address the needs of youth and families (Bruns et al. 2006).

The purpose of our study was to examine the relationships among the implementation of necessary support conditions for wraparound, wraparound fidelity and youth outcomes. The local evaluation of a grant authorized by the Centers for Medicare and Medicaid Services to demonstrate that intensive community based services could be a viable alternative to psychiatric residential treatment facilities (PRTF; George 2010) provided the data necessary to explore these relationships. A site assessment was used to measure the stage of development (Rogers 2003; Fixsen et al. 2005; Glisson and Schoenwald 2005) that each community in Indiana achieved in terms of implementing the necessary conditions for wraparound (Walker et al. 2003). Wraparound fidelity was measured and examined in relationship to a community's stage of development and to outcomes observed for youth and families. Outcomes for youth were measured with the Child and Adolescent Needs and Strengths (CANS; Lyons 2009). We hypothesized that communities in later stages would have higher fidelity to wraparound than communities at earlier stages and that youth receiving high fidelity wraparound would have better outcomes than youth not receiving wraparound or experiencing wraparound with lower levels of fidelity.

\section{Method}

\section{Participants}

Participants in this study included youth who received intensive community-based services through the Community Alternatives to Psychiatric Residential Treatment Facilities (CA-PRTF) Medicaid Demonstration grant in Indiana between January 2008 and June 2010. Youth served have severe emotional and behavioral health needs as well as functional impairments that might otherwise be treated in a PRTF. Study participants were limited to youth for whom site assessment, wraparound fidelity, and outcome data were available.

\section{Dependent Variables and Procedures}

Stage of Development. The Strengths-Based Site Assessment (Effland 2009) measures the extent to which the following organizational and systemlevel support conditions for effective wraparound are in place within each community (Walker et al. 2003): collaboration and partnerships (representation by child-serving agencies and families); capacity building and staffing (project staff, strengthbased supervision); acquiring services and supports (funding sources, Medicaid billing, flexible funds); accountability (outcome measurement and reporting); and family involvement.

During regular visits to Indiana communities, coaches employed by a technical assistance and training center dedicated to supporting the implementation of wraparound (Choices 2010) rate the level of development in each of five support conditions. The ratings are based on change theories that identify five stages through which individuals (Prochaska et al. 1994) and organizations (Rogers 2003) move as they contemplate and implement change. The five stages were consolidated into four stages for this study because of the small number of sites rated in the first two stages (i.e., precontemplation and contemplation). An overall stage of development was determined for each community by averaging the ratings assigned across support conditions and identifying the closest stage.

Wraparound Fidelity. The Wraparound Fidelity Index 4.0 (WFI; Bruns et al. 2007) measures the 
extent to which communities apply the ten wraparound principles identified adopted by the NWI (Miles et al. 2006) in services to youth and families The ten principles of wraparound became the ten subscales of the WFI with four questions reflect each principle. Table 1 describes each wraparound principle, WFI domain.

The WFI 4.0 and earlier versions of the tool are both reliable and valid (Bruns et al. 2004, 2007). WFI interviewers complete a comprehensive training protocol that includes certification to insure that WFI ratings are made reliably. The WFI was administered through telephone interviews with wrap- around facilitators at multiple times (i.e., approximately 3 months after grant services began, 1 year after the beginning of grant services and within 30 days of discharge) during a youth's involvement in the grant. In order to attain sufficient sample sizes, the most recent interview completed for each youth was included in the analysis. As a result, some youth had been discharged from grant services, while others had been involved for 12 months or less.

Interviewers assigned a score to each question on a scale of 0 (low fidelity) to 2 (high fidelity). WFI scores were calculated by summing across items rated on the WFI and dividing by the highest pos-

Table 1. Wraparound Fidelity Index subscales: principles of wraparound (Bruns et al. 2008)

\begin{tabular}{|c|c|}
\hline Principles & Description \\
\hline $\begin{array}{l}\text { 1. Family voice and } \\
\text { choice }\end{array}$ & $\begin{array}{l}\text { Family and child perspectives are elicited and prioritized in all phases of the wraparound } \\
\text { process. Planning is grounded in family perspectives, and the team strives to provide } \\
\text { options so that the plan reflects family values and preferences }\end{array}$ \\
\hline 2. Team based & $\begin{array}{l}\text { The wraparound team is made up of individuals agreed upon by and committed to the } \\
\text { family }\end{array}$ \\
\hline 3. Natural supports & $\begin{array}{l}\text { The team seeks out and encourages the participation of members from family members' } \\
\text { relationships. The plan includes activities and intervention involving natural support }\end{array}$ \\
\hline 4. Collaboration & $\begin{array}{l}\text { Team members cooperate and share responsibility for developing, implementing, } \\
\text { monitoring, and evaluating a single plan. The plan blends team members' perspectives, } \\
\text { mandates, and resources. Each team member's work is guided by the plan }\end{array}$ \\
\hline 5. Community based & $\begin{array}{l}\text { The wraparound team implements service and support strategies that take place in the } \\
\text { most inclusive, most responsive, most accessible, and least restrictive settings possible, } \\
\text { that safely promote child and family integration into home and community life }\end{array}$ \\
\hline $\begin{array}{l}\text { 6. Culturally } \\
\text { competent }\end{array}$ & $\begin{array}{l}\text { The wraparound process demonstrates respect for and builds on the values, preferences, } \\
\text { beliefs, culture, and identity of the child, family, and their community }\end{array}$ \\
\hline 7. Individualized & $\begin{array}{l}\text { To achieve the goals and objectives in the wraparound plan, the team develops and } \\
\text { implements a tailored set of supports, and services }\end{array}$ \\
\hline 8. Strengths based & $\begin{array}{l}\text { The wraparound process and the wraparound plan identify, build on, and develop the } \\
\text { capabilities, knowledge, skills, and assets }\end{array}$ \\
\hline 9. Unconditional & $\begin{array}{l}\text { A wraparound team does not give up on, blame, or reject youth, and their families. When } \\
\text { faced with challenges or a setback, the team continues working towards meeting the } \\
\text { needs of the youth and family and towards achieving the plan goals until the team agrees } \\
\text { that a formal wraparound process is no longer necessary }\end{array}$ \\
\hline 10. Outcomes based & $\begin{array}{l}\text { The team links the youth and family's goals of the wraparound plan to address identified } \\
\text { needs and support or build strengths, uses observable or measurable objectives to } \\
\text { monitor progress, and revises the plan to address changes }\end{array}$ \\
\hline
\end{tabular}


sible fidelity score. For descriptive analyses, total fidelity scores from facilitators were assigned to one of four categories based on established fidelity benchmarks (Bruns et al. 2005): low wraparound fidelity (scores below 65\%); borderline (scores between 65 and 74\%); adequate (scores between 75 and $84 \%$ ); and high (scores of $85 \%$ and above). For predictive analyses, raw fidelity scores were used.

Outcomes. In 2007, Indiana adopted the CANS as the outcome management tool for the behavioral health service system. The CANS has been found to be reliable and valid (Lyons 2009). An online training and certification process allows individuals throughout Indiana to become certified users of the CANS. The average certification reliability for Indiana's 4300 certified CANS users is .79, consistent with Anderson et al. (2003). Local support for rating and using the CANS in the treatment planning process is provided by SuperUsers who receive advanced training on the CANS. All wraparound facilitators have become SuperUsers with a minimal certification reliability of .75 .

Indiana's comprehensive multisystem CANS tool includes the following dimensions: child strengths, functioning, behavioral/emotional needs, risk behaviors, and caregiver strengths and needs. CANS items are rated using a four-point scale (0-3) to indicate whether identified needs require action and whether strengths can be used in treatment planning. CANS assessments are completed several times during a specific episode of service: baseline, every 6 months, and at discharge or when a change in the level of care is being considered. The CANS tool includes six dimensions (functioning needs, behavioral/emotional needs, risk behavioral needs, youth strengths, caregivers' strengths and needs and acculturation). Ratings from three CANS dimensions (i.e., functioning, behavioral/emotional needs and risk behaviors) were averaged to create a youth needs domain. This scoring strategy allows researchers to use a single score to represent the functional status of a youth (Lyons 2009). The strategy is supported by Doucette's (2007) scaling of the comprehensive version of the CANS. Functional improvement was then defined as any decrease in youth needs from the baseline assessment to the most recent CANS assessment. For this study, only change in youth needs was measured.

Relationship Between Stage of Development and Wraparound Fidelity. To better understand the relationship between necessary organizational and system-level support conditions and fidelity to the wraparound, the data was categorized into four stages of development (i.e., precontemplation/ contemplation, preparation, action and maintenance) and four levels of wraparound fidelity (not wraparound, borderline, adequate and high fidelity wraparound). An ANOVA was used to determine if significant associations existed.

Predicting Improvement. To determine whether variables that predict positive outcomes for youth could be identified, two hierarchical regression models were run using a forced entry method in SPSS (2010). In the first model, the following independent variables were included as possible predictors of change in youth needs: total facilitator WFI fidelity score, stage of development, baseline functioning from CANS domains (behavioral health, functioning, risks, strengths and caregiver strengths and needs), age, gender, race (Black/African American, Native American, multi-racial) and ethnicity (Hispanic). In the second regression model (Model 2), the high level, aggregated predictors were deconstructed to better understand what components of the service delivery model and which behavioral health needs are related to outcomes. Baseline behavioral health CANS items ratings were substituted for baseline behavioral health domain scores and facilitators' ten WFI subscales replaced the facilitator Total WFI scores. Specifically, Step 1 began with the ten elements of wraparound. Step 2 included baseline behavioral health needs from the CANS (psychosis, impulsivity, depression, anxiety, oppositional, conduct, adjustment to trauma, anger control, substance use and eating disorder). Step 3 retained other baseline CANS domains (functioning, risks, strengths and caregiver) which were subsequently deconstructed. From youth demographic characteristics, Step 4 retained the predictive racial item. For each model, non-significant factors were removed. 


\section{Results}

\section{Participants}

This study includes 515 youth who received services through the CA-PRTF grant between Jan 2008 and June 2010. Most of these youth were male (72.6\%); $77.3 \%$ were Caucasian, $16.1 \%$ were African American, $1.2 \%$ were Native American, 5.4\% identified themselves as being of two or more races; and $3.7 \%$ were Hispanic. Youth ranged from 6 to 20 years old $(M=13.67, S D=3.1)$.

\section{Stage of Development}

TA Center coaches assessed 65 communities between December 2008 and May 2010 using the Strengths-Based Site Assessment (Effland 2009). The majority of communities were evaluated as being at Stages $2(28.8 \%)$ and $3(34.8 \%)$ at their most recent assessment; $15.2 \%$ of sites were evaluated as being at Stage 1 and $19.7 \%$ in Stage 4 .

\section{Wraparound Fidelity}

The average overall facilitator WFI score was $80.7(S D=.10)$. Of the 515 participants, $41.3 \%$ received high fidelity wraparound, $32.6 \%$ were receiving an adequate level of wraparound, 15.5\% were receiving wraparound at a borderline level of fidelity, and $7.1 \%$ did not receive wraparound.

\section{Stage of Development and Wraparound Fidelity}

WFI interviews were completed for at least one youth in 46 of the 65 communities that also had a completed site assessment. Specifically, 56 youth were interviewed in 7 communities in Stage 1, 63 youth were interviewed in 13 communities in Stage 2,309 youth were interviewed in 17 communities in Stage 3, and 79 youth were interviewed in 15 communities in Stage 4.

To examine the relationship between a community's stage of development and the level of wraparound fidelity, the percent of youth that received wraparound at each of the four fidelity levels was computed for each of the four stages of implementation. The results indicate that a higher percent of respondents $(46.4 \%)$ reported receiving high fidelity wraparound in communities in Stage
1 than in communities at the remaining three stages (i.e., $25.4 \%$ at Stage 2, $49.7 \%$ at Stage 3 and $38.1 \%$ at Stage 4). A higher percent of respondents reported an adequate level of wraparound fidelity in Stage $4(34.0 \%)$ than in Stage 1 (32.1\%), Stage 2 $(30.2 \%)$ or Stage $3(32.4 \%)$. Approximately $23.8 \%$ of respondents in Stage 2 communities reported a borderline level of fidelity, compared to $16.1 \%$ of respondents in Stage 1 communities, $12.1 \%$ in Stage 3 communities and $15.3 \%$ in Stage 4 communities. The percent of respondents that indicated that they did not receive wraparound was highest in Stage 2 communities (14.3\%), followed by Stage 4 (9.3\%), Stage $1(3.6 \%)$ and Stage $3(2.9 \%)$.

There was a significant association between stage of development and the level of wraparound fidelity as reported by facilitators. Figure 1 graphically describes the frequency of different levels of wraparound fidelity across stages of site development. A significant association was found between the with community stage of development and levels of fidelity, $F(3,5.72), p<.01, w=.2$. There is a significant cubic trend, $F(3,506)=21.449, p<.001$, $w=.20$. Figure 2 depicts the directional change in mean levels of fidelity among sites. Specifically, planned contrasts revealed a significant relationship between earlier (contemplation and preparation) and later (action and maintenance) stages of site development, $t(156.57)=2.28, p<.05, r=.168$.

\section{Outcomes}

Change in youth needs is operationally defined as the difference between youth needs (i.e., ratings across the behavioral/emotional, functioning and risks domains) at the most recent assessment minus baseline youth needs. Given the item scoring, a negative change score indicates improvement. Of the 515 youth included in the analysis, $60.7 \%$ experienced reduced needs.

\section{Predicting Improvement}

Table 2 reports means, standard deviations, and inter-correlations for dependent variable and the seven significant predictor variables in the first regression model (Model 1) for 515 youth. Since reduction in youth needs is indicated by a nega- 
Figure 1. Relationship between site stage of development and level of wraparound fidelity

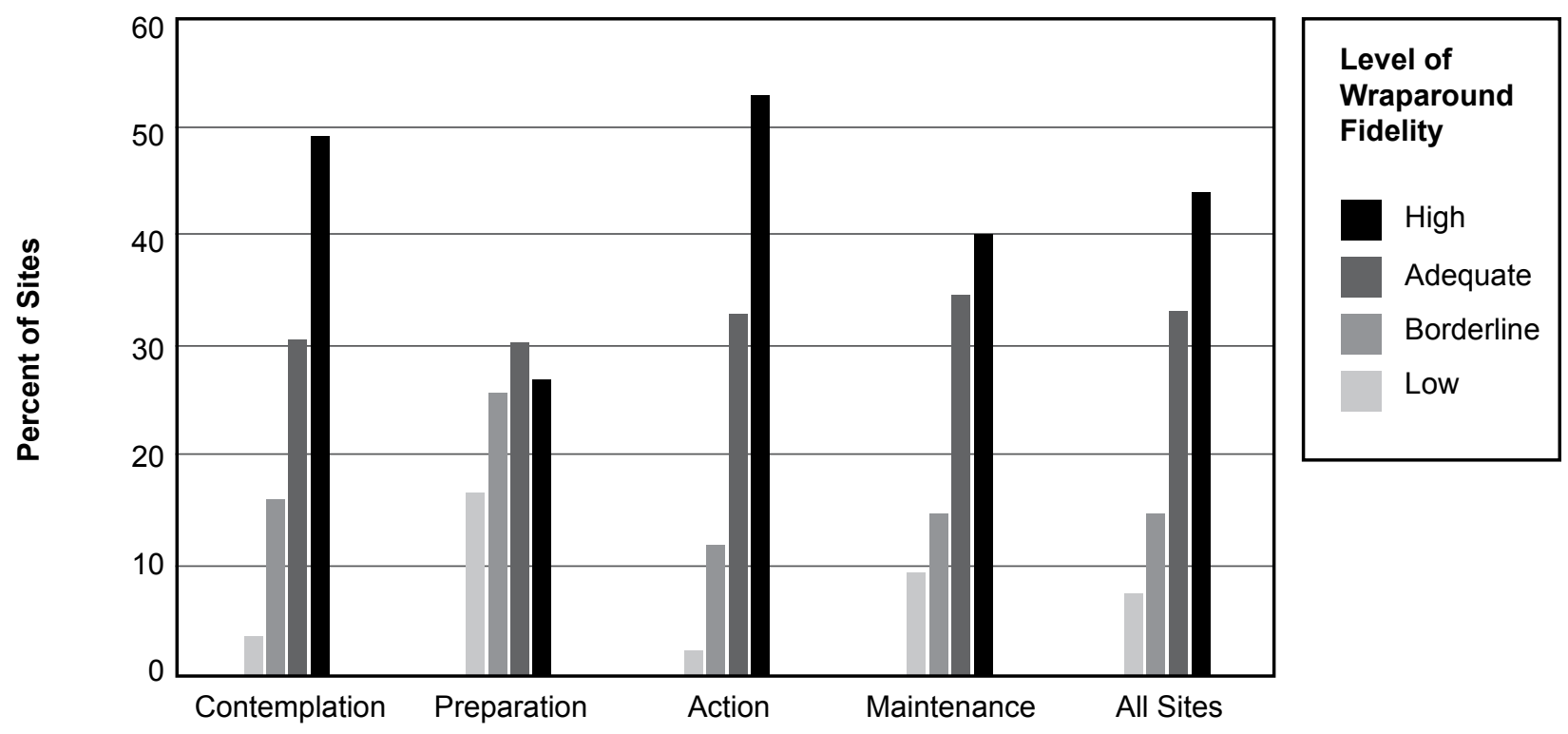

Site Stage of Development

Figure 2. Mean wraparound fidelity score for sites at each stage of development

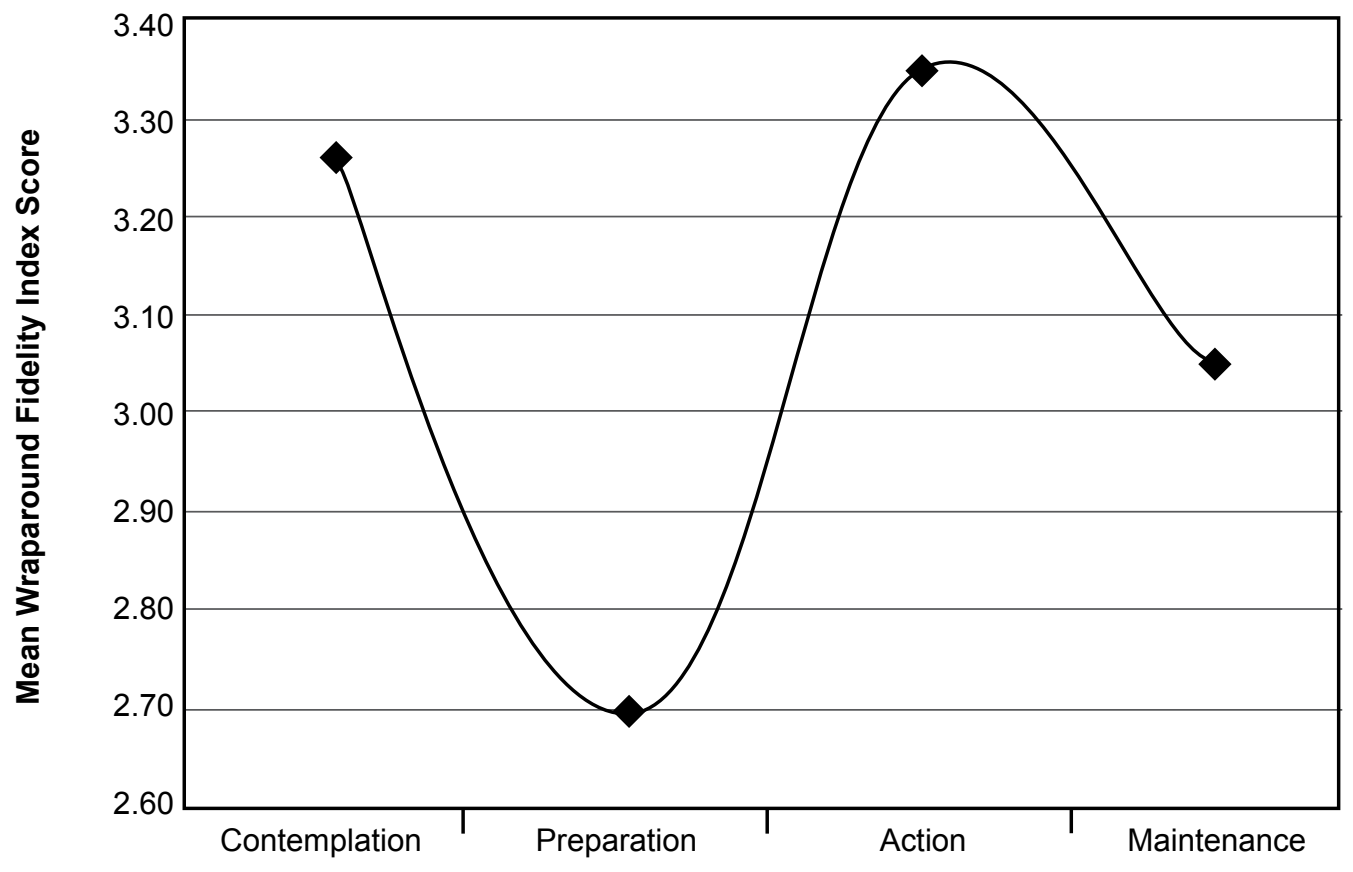

Site Stage of Development 
tive change score (i.e., decreasing scores indicate decreased needs), negative standardized beta scores, $\beta$, indicate a positive relationship between higher predictive values for fidelity and baseline CANS ratings and improved youth's needs. For example, the mean youth change $(\beta=-1.59, S D=4.16)$, reflects an overall improvement on the 0-3 CANS scale.

In Model 1, significant correlations exist between change in youth needs and each remaining predictive variable. The strongest correlations between dependent and predictive variables were between change in youth needs and baseline behavioral health needs $(r=.38, p<.001)$ and change in youth needs and baseline functioning ( $r=.38$, $p<.001)$. The strongest inter-correlation between predictors was between baseline behavioral health needs and risks $(r=.49, p<.001)$ and functioning $(r=.44, p<.001)$, respectively. The correlation between the fidelity score and change is significant, but relatively small $(r=-.13, p<.01)$.
Total wraparound fidelity has a significant, but small impact on improvement $(\beta=-.14, p<.001)$ when baseline functioning, gender, age, race and ethnicity are held constant (see Table 3). The strongest predictors of improvement are baseline needs: behavioral health $(\beta=-.18, p<.001)$, functioning $(\beta=-.18, p<.001)$ and risks $(\beta=-.16, p<.001)$. Youth who identify themselves as being of two or more racial groups are less likely to benefit $(\beta=.10$, $p<.001)$ from wraparound. Overall, the model's predictors account for $26.7 \%$ of the variability in change in youth needs.

The results of the second regression model, reported in Tables 4 and $5(n=498)$, indicate specific significant elements of the wraparound services model and baseline needs. Some youth were excluded from the analysis when individual fidelity and CANS items were predictors. The final model included 15 predictive factors, 14 of which were components of wraparound fidelity or baseline

Table 2. Means, standard deviations, and intercorrelations for dependent and predictor variables, Model 1

\begin{tabular}{|c|c|c|c|c|c|c|c|c|c|}
\hline Variable & $\mathbf{M}$ & SD & 1 & 2 & 3 & 4 & 5 & 6 & 7 \\
\hline $\begin{array}{l}\text { Change in youth } \\
\text { needs }\end{array}$ & -1.59 & 4.16 & $-.13^{* *}$ & $-.38^{\star \star *}$ & $-.38^{\star * *}$ & $-.26^{\star \star \star}$ & $-.24^{\star \star \star}$ & $-.34^{* * *}$ & $.12^{* *}$ \\
\hline \multicolumn{10}{|l|}{ Predictor variables } \\
\hline $\begin{array}{l}\text { 1. Total facilitator } \\
\text { WFI score }\end{array}$ & .81 & .10 & - & .01 & -.03 & -.01 & .03 & -.05 & -.02 \\
\hline $\begin{array}{l}\text { 2. Baseline } \\
\text { behavioral health }\end{array}$ & 15.96 & 3.32 & & - & $.45^{\star \star *}$ & $.25^{* * *}$ & $.17^{\star \star *}$ & $.49^{* * *}$ & .01 \\
\hline $\begin{array}{l}\text { 3. Baseline } \\
\text { functioning }\end{array}$ & 15.38 & 3.70 & & & - & $.35^{\star \star *}$ & $.29^{\star \star *}$ & $.35^{\star \star \star}$ & $-.08^{*}$ \\
\hline $\begin{array}{l}\text { 4. Baseline child } \\
\text { strengths }\end{array}$ & 19.93 & 4.27 & & & & - & $.30^{* * *}$ & $.17^{\star * *}$ & .02 \\
\hline $\begin{array}{l}\text { 5. Baseline caregiver } \\
\text { domain }\end{array}$ & 12.27 & 4.40 & & & & & - & $.17^{\star \star \star}$ & -.06 \\
\hline 6. Baseline risks & 11.76 & 3.21 & & & & & & - & -.03 \\
\hline 7. Multi-racial & .04 & .23 & & & & & & & - \\
\hline
\end{tabular}

${ }^{\star} p<0.05 ;{ }^{* *} p<0.01 ;{ }^{* *} p<.001 ; N=515$ 
needs and strengths plus youth with mixed racial identity.

The strongest correlation between the dependent variable and the predictor variables was found between change in youth needs and baseline conduct behavioral needs $(r=-.276, p<.001)$, followed closely by the outcomes based element of wraparound $(r=-.244, p<.001)$, bullying $(r=$ $-.242, p<.001)$, functioning at home $(r=-.217, p<$ $.001)$ and functioning at school $(r=-.207, p<.001)$. Among predictors, the strongest correlation was found between baseline conduct and delinquency $(r=.314, p<.001)$.
Among the strongest predictors of improvement for youth were the outcomes based $(\beta=-5.87$, $p<.001)$ and the community based $(\beta=-2.47, p$ $<.001)$ elements of wraparound fidelity. Unexpectedly, the cultural competency element $(\beta=5.09, p<$ $.01)$ predicted increased needs, with $95 \%$ of wraparound facilitators reporting that the wraparound teams met cultural fidelity requirements. Specific baseline needs and strengths made relatively small contributions to positive change. Youth who identified themselves as being multi-racial were less likely than Caucasian youth to improve $(\beta=1.76, p<.05)$.

Table 3. Hierarchical regression model predicting change in youth needs, Model $1(N=515)$

\begin{tabular}{|c|c|c|c|c|c|}
\hline Variable & $B$ & SEB & $\beta$ & $R^{2}$ & $\Delta R^{2}$ \\
\hline Step 1 & & & & .017 & .017 \\
\hline Facilitator total WFI score & -5.540 & 1.843 & $-.132^{* *}$ & & \\
\hline Step 2 & & & & .256 & .239 \\
\hline Facilitator total WFI score & -5.917 & 1.616 & $-.141^{* \star *}$ & & \\
\hline Baseline behavioral health & -.222 & .059 & $-.177^{* * *}$ & & \\
\hline Baseline functioning & -.218 & .051 & $-.195^{* * *}$ & & \\
\hline Baseline risks & -.204 & .058 & $-.157^{\star \star *}$ & & \\
\hline Baseline strengths & -.088 & .041 & $-.091^{*}$ & & \\
\hline Baseline caregiver & -.081 & .039 & $-.086^{\star}$ & & \\
\hline Step 3 & & & & .267 & .010 \\
\hline Facilitator total WFI score & -5.838 & 1.607 & $-.139^{\star \star \star}$ & & \\
\hline Baseline behavioral health & -.230 & .059 & $-.183^{* * *}$ & & \\
\hline Baseline functioning & -.206 & .051 & $-.195^{\star \star \star}$ & & \\
\hline Baseline risks & -.201 & .058 & $-.155^{\star \star}$ & & \\
\hline Baseline strengths & -.094 & .041 & $-.097^{*}$ & & \\
\hline Baseline caregiver & -.076 & .038 & $-.081^{*}$ & & \\
\hline Multi-racial & 1.847 & .701 & $.101^{* *}$ & & \\
\hline
\end{tabular}

${ }^{\star} p<0.05 ;{ }^{* *} p<0.01 ;{ }^{* *} p<.001$ 


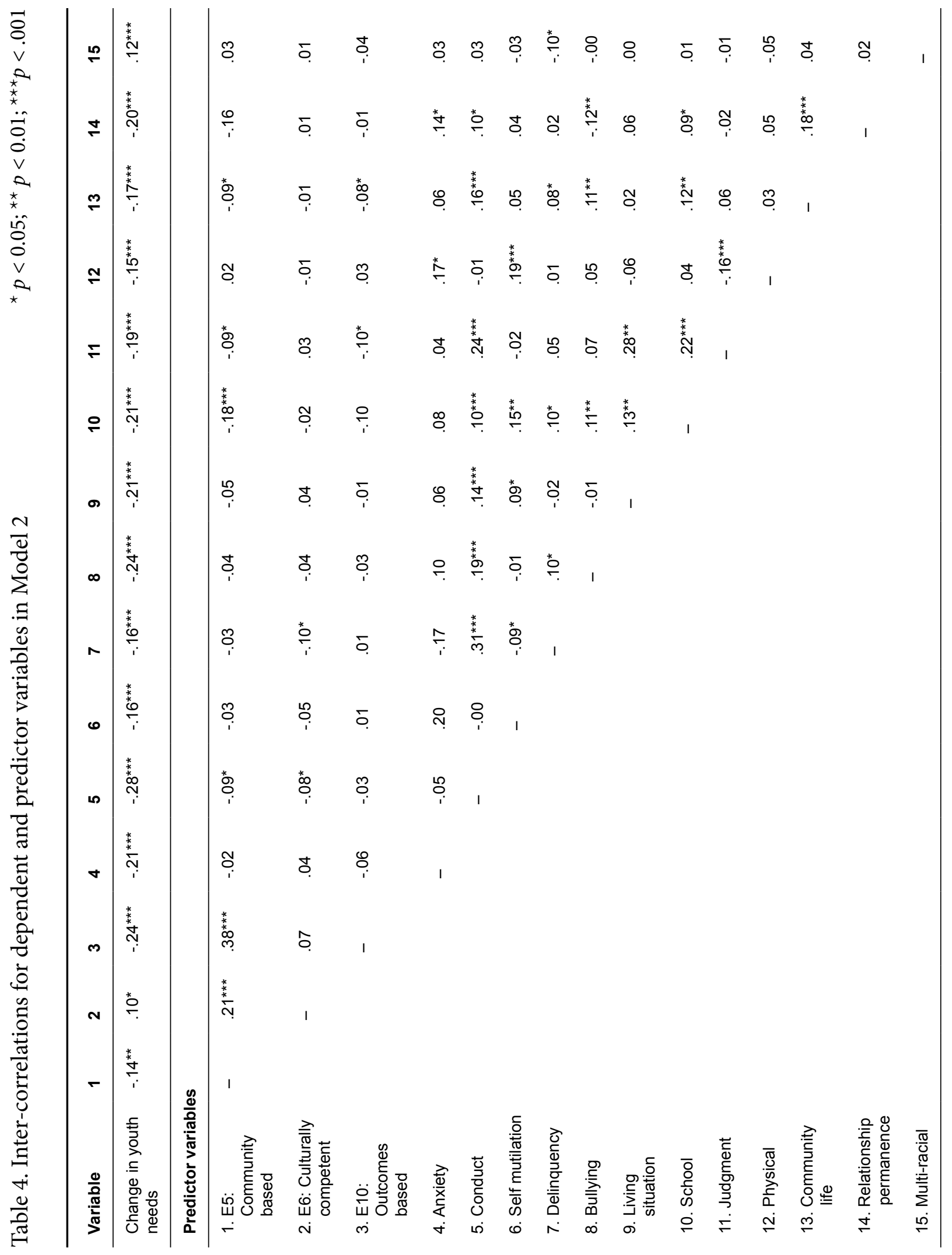


Table 5. Regression model predicting change in youth needs, Model $2(n=498)$

\begin{tabular}{|c|c|c|c|c|c|}
\hline Variable & $B$ & SEB & $\beta$ & $R^{2}$ & $\Delta R^{2}$ \\
\hline Step 1 & & & & $.076^{* * *}$ & \\
\hline E5: Community based & -1.32 & .82 & -.08 & & \\
\hline E6: Culturally competent & 5.03 & 1.73 & $.13^{* *}$ & & \\
\hline E10: Outcomes based & -5.43 & 1.16 & $-.22^{\star \star *}$ & & \\
\hline Step 2 & & & & $.36^{\star * \star}$ & .283 \\
\hline E5: Community based & -2.40 & .70 & $-.14^{\star *}$ & & \\
\hline E6: Culturally competent & 5.08 & 1.47 & $.13^{\star \star}$ & & \\
\hline E10: Outcomes based & -5.98 & .98 & $-.24^{\star \star *}$ & & \\
\hline Anxiety & -.78 & .18 & $-.17^{* * *}$ & & \\
\hline Conduct & -.73 & .19 & $-.15^{\star \star *}$ & & \\
\hline Self mutilation & -.38 & .17 & $-.08^{*}$ & & \\
\hline Delinquency & -.34 & .14 & $-.10^{*}$ & & \\
\hline Bullying & -.56 & .14 & $-.15^{\star * *}$ & & \\
\hline Living situation & -.77 & .21 & $-.14^{\star * *}$ & & \\
\hline School & -.62 & .21 & $-.11^{* *}$ & & \\
\hline Judgment & -.64 & .22 & $-.12^{\star *}$ & & \\
\hline Physical & -.63 & .24 & $-.10^{\star *}$ & & \\
\hline Community life & -.48 & .20 & $-.09^{*}$ & & \\
\hline Relationship permanence & -.55 & .21 & $-.10^{*}$ & & \\
\hline Step 3 & & & & $.37^{\star \star \star}$ & .009 \\
\hline E5: Community based & -2.47 & .70 & $-.14^{* * *}$ & & \\
\hline E6: Culturally competent & 5.09 & 1.46 & $.13^{* *}$ & & \\
\hline E10: Outcomes based & -5.87 & .98 & $-.24^{* * *}$ & & \\
\hline Anxiety & -.76 & .18 & $-.17^{* * *}$ & & \\
\hline Conduct & -.73 & .19 & $-.15^{\star * *}$ & & \\
\hline Self mutilation & -.36 & .17 & $-.08^{*}$ & & \\
\hline Delinquency & -.30 & .14 & $-.09^{*}$ & & \\
\hline Bullying & -.56 & .14 & $-.15^{\star \star *}$ & & \\
\hline Living situation & -.77 & .21 & $-.14^{\star \star \star}$ & & \\
\hline School & -.62 & .21 & $-.12^{\star *}$ & & \\
\hline Judgment & -.64 & .22 & $-.12^{\star *}$ & & \\
\hline Physical & -.60 & .24 & $-.10^{*}$ & & \\
\hline Community life & -.51 & .20 & $-.10^{*}$ & & \\
\hline Relationship permanence & -.55 & .21 & $-.10^{\star *}$ & & \\
\hline Multi-racial & 1.76 & .68 & $.10^{*}$ & & \\
\hline
\end{tabular}

${ }^{\star} p<0.05 ;{ }^{* *} p<0.01 ;{ }^{* *} p<.001$ 


\section{Discussion}

The descriptive analysis conducted in this study suggests that there is a significant relationship between the implementation of several system and organizational components and wraparound fidelity. Specifically, high fidelity was reported more often by facilitators in communities at Stages 3 and 4 of implementation than in Stage 2 communities. Unexpectedly, nearly $46.4 \%$ of facilitators in Stage 1 communities also reported a high level of fidelity. When interpreting this result, it is important to remember that only seven communities were included in Stage 1 compared to over 13 communities in each of the remaining three stages. Additionally, the absence of necessary system and organizational conditions to support wraparound at the local level resulted in grant representatives spending extra time in these communities and infusing a higher level of support and coaching of wraparound facilitators and supervisors than was provided elsewhere. The grant's policies, procedures and quality improvement initiatives may be compensating for missing strong stable local organizational and system support necessary for high quality collaborative, individualized service planning, support and effective services (Walker et al. 2003). Thus, the level of wraparound fidelity observed in Stage 1 is likely the function of state-level support for wraparound rather than the presence of necessary support conditions in the local community. Measuring the level of state team monitoring and support given to local providers is indicated for future studies.

The results of this study indicate that although stage of development is significantly associated with the level of wraparound fidelity, it is not predictive of improvement in youth needs. This result can be partially explained by the different methods used in this study. The descriptive analysis compared the level of association between the stage of development and the level of wraparound fidelity. The regression analysis used the average implementation score across the necessary system and organizational support conditions assessed and the total WFI score from interviews with facilitators. By doing so, the relationship between stage of development and both wraparound fidelity and youth outcomes diminishes. Further research is needed to determine if lack of inter-rater reliability of the site assessment used in this study contributed to this pattern of results.

An association between high fidelity wraparound and improvement in youth needs was supported by the regression correlations. The initial regression model includes composite dimensions, which support the relevance of fidelity to wraparound, but are difficult to interpret. Consistent with other studies (e.g., Cox et al. 2010; McGrew et al. 1994), the components of total fidelity scores, baseline needs and strengths not only explain more of the variability in the change in youth needs, but add meaning to the findings as well. While all of the elements of the wraparound services model are important to the integrity of the model, not all components may be related to outcomes for youth.

Although the specific predictors in this study differ from other findings (Cox et al. 2010), the importance of involvement in community based activities, is reinforced by the predictive relationship between community-based services and improvement in youth functioning. The current study also suggests that adherence to the outcomes-based element of wraparound contributes to decreased needs for youth, when holding baseline functioning and demographic variables (i.e., age, gender, race and ethnicity) constant. In other words, evaluating and linking needs and strengths to the intervention plan, actively monitoring progress and making changes is important to improvement.

The unanticipated and incongruent finding that cultural competency predicts worsening of needs may reflect rating issues for this fidelity element. Specifically, about $95 \%$ of facilitators reported that wraparound was implemented with fidelity to the cultural competence element. Future research that includes WFI interviews with caregivers and youth is needed to determine whether wraparound was actually implemented with this high level of fidelity to this element or if the ratings of the wraparound facilitators were somewhat biased.

The importance of specific baseline needs and strengths and race, help answer the important question of who benefits from wraparound. Youth with high levels of anxiety conduct disorders, delinquent 
and bullying behaviors and who demonstrate functional problems at home, at school and in the community were more likely to benefit from wraparound than youth who did not have needs in these areas. These findings reinforce previous assumptions that the model is better suited for youth with some internalized behavioral health needs, behavioral issues, functional impairments and disruptions in family relationships. Absent are findings related to high levels of depression, impulse control or psychosis.

\section{Limitations}

Several limitations of the current study should be noted. First, the data for this study came from the evaluation of Indiana's CA-PRTF grant. The communities and youth represented in this study may not be comparable to youth participating in this demonstration grant in other states or to youth who receive wraparound that is supported by other funding mechanisms. Additional research is needed to determine the extent to which these findings will generalize to other populations of youth.

Second, the distribution of youth served in communities across the four stages of implementation was not equivalent, with nearly $60.9 \%$ of the youth represented in this study served in Stage 3 (i.e., action). Similarly, the overall WFI score for the youth included in this study was $80.7 \%$ and the majority $(73.9 \%)$ of youth received wraparound at adequate or high levels of fidelity. These results suggest that wraparound has been successfully implemented in Indiana, but may have limited our ability to fully understand the relationships that exist among stage of development, wraparound fidelity and outcomes. Future research should include one or more comparison groups of similar youth receiving usual public behavioral health services and/ or youth receiving wraparound at lower levels of fidelity.

Third, although WFI interviewers and CANS raters were required to achieve minimum reliability requirements in order to use the tools, similar requirements do not exist for the stage of development ratings made using the Strengths-Based Site Assessment. A method to assess inter-rater reliability for the site assessment needs to be developed and included in future studies. Fourth, a measure of the level of state oversight provided to communities at each stage of development is needed in order to help explain the unexpected finding that youth in Stage 1 communities experienced high level of wraparound fidelity. Fifth, the complexity of the issues examined limited the conclusions which could be reached from the data used in this study. For example, the outcome measure used (i.e., change in youth needs) is a composite rating of behavioral health needs, functioning and risk behaviors. This composite score lacks details about specific areas of change that would help clarify how the identified predictor variables contribute to overall improvements in functioning.

Sixth, measurement of wraparound fidelity in this study was limited to WFI interviews conducted with facilitators and not caregivers or youth. This is a significant limitation of the study because the families' experiences of wraparound could have differed substantially from that of the facilitator. Finally, the wraparound interviews included in this study were the most recent interviews available for each youth, rather than interviews conducted at a specified time (e.g., 6 months after starting wraparound or at the completion of wraparound). As future interviews are completed, especially for youth who have completed the wraparound process, the results of this study should be re-examined.

\section{Conclusions}

The results of our study support the hypothesized relationship between (1) stage of development of necessary system level support conditions for wraparound and wraparound fidelity and (2) wraparound fidelity and youth outcomes. Additionally, this study identifies several variables that predict improvement in youth functioning within the wraparound process. Specifically, the results suggest that when wraparound is implemented with high fidelity to the outcomes-based and communitybased elements, youth are more likely to improve. Wraparound is most beneficial to youth with high levels of anxiety, behavioral disorders (conduct disorders, delinquency or bullying) and low functioning at home, school and in the community. Interestingly, community stage of development was not directly associated with youth outcomes in this 
study. The fact that stage of development was significantly associated with high fidelity to wraparound, however, suggests that researchers should continue to refine tools to measure system level conditions supporting wraparound.

The relationship between wraparound fidelity, youth strengths, and functional outcomes also deserves further investigation. The continuing expansion of wraparound in Indiana will provide an opportunity to examine some of these issues. Not only will the CA-PRTF grant continue to provide information on wraparound implementation, the expansion of wraparound into other child-serving systems (e.g., child welfare) will provide additional sources of information and opportunities for comparison. The lessons learned from this study should help leaders in the behavioral health system keep focused on the importance of effectively implementing the necessary support conditions for wraparound and supporting high fidelity wraparound for youth and their families.

\section{References}

Anderson, R. L., Lyons, J. S., Giles, D. M., Price, J. A., \& Estes, G. (2003). Examining the reliability of the Child and Adolescent Needs and Strengths-Mental Health (CANS-MH) Scale from two perspectives: A comparison of clinician and researcher ratings. Journal of Child and Family Studies, 12, 279-289.

Bertram, R. M., Suter, J. C., Bruns, E. J., \& O’Rourke, K. E. (2010). Implementation research and wraparound literature: Building a research agenda. Journal of Child and Family Studies. doi:10.1007/s10826-010-9430-3.

Bruns, E. J., Burchard, J. D., Suter, J. C., Leverentz-Brady, K., \& Force, M. M. (2004). Assessing fidelity to a community-based treatment for youth: The Wraparound Fidelity Index. Journal of Emotional and Behavioral Disorders, 12(2), 79-89.

Bruns, E. J., Leverentz-Brady, K. M., \& Suter, J. C. (2005). Is it wraparound yet? Determining fidelity standards for the wraparound fidelity index. In C. Newman, C. Liberton, K. Kutash, \& R. M. Friedman (Eds.), The 18th annual proceedings, a system of care for children's mental health: Expanding the research base (pp. 185-190). Tampa, FL: University of South Florida, The Louis de la Parte Florida Mental Health Institute, Research and Training Center for Children's Mental Health.

Bruns, E. J., Rast, J., Peterson, C., Walker, J., \& Bosworth, J. (2006a). Spreadsheets, service providers, and the statehouse: Using data and the wraparound process to reform systems for children and families. America Journal of Community Psychology, 38, 201-212.

Bruns, E. J., \& Suter, J. C. (2010). Summary of the wraparound evidence base. In E. J. Bruns \& J. S. Walker (Eds.), The resource guide to wraparound. Portland, OR: National Wraparound Initiative.

Bruns, E., Suter, J., Force, M., Sather, A., \& Leverentz-Brady, K. (2007). Wraparound fidelity index 4.0: Manual for training, administration, and scoring of the WFI 4.0, August 13, 2007 version. Seattle, WA: Wraparound Evaluation and Research Team, Department of Psychiatry \& Behavioral Sciences, University of Washington.

Bruns, E. J., Suter, J. C., \& Leverentz-Brady, K. M. (2006b). Relations between program and system variables and fidelity to the wraparound process for children and families. Psychiatric Services, 57, 1586-1593.

Bruns, E. J., Walker, J. S., \& The National Wraparound Initiative Advisory Group. (2008). Ten principles of the wraparound process. In E. J. Bruns \& J. S. Walker (Eds.), The resource guide to wraparound. Portland, OR: National Wraparound Initiative, Research and Training Center for Family Support and Children's Mental Health.

Bruns, E. J., Walker, J. S., Zabel, M., Matarese, M., Estep, K., Harburger, D., et al. (2010). Intervening in the lives of youth with complex behavioral health challenges and their families: The role of the wraparound process. American Journal of Community Psychology, 46, 314-331.

Choices, Inc. (2010). Training and consultation. Retrieved November 6, 2010 from http://www.choicesteam.org/ tacenter.html.

Cox, K., Baker, D., \& Wong, M. A. (2010). Wraparound retrospective: Factors predicting positive outcomes. Journal of Emotional and Behavioral Disorders, 18, 3-13.

Doucette, A. (2007). Measurement properties: Child and adolescent needs and strengths (CANS). 4th annual CANS conference, Boston, October.

Effland, V. S. (2009). Monthly site assessment rating guide. Indianapolis, IN: Choices TA Center, Choices, Inc.

Fixsen, D. L., Naoom, S. F., Blasé, K. A., Friedman, R. M., \& Wallace, F. (2005). Implementation research: A synthesis of the literature. Tampa, FL: University of South Florida, Louis de la Parte Florida Mental Health Institute, The National Implementation Research Network (FMHI Publication \#231).

George, E. R. (2010). Community-based alternatives to PRTF. Presentation at the 23rd annual children's mental health research \& policy conference, March 2010. Tampa, FL: University of South Florida. 
Glisson, C., \& Schoenwald, S. K. (2005). The ARC organizational and community intervention strategy for implementing evidence-based children's mental health treatments. Mental Health Service Research, 70(4), 243-259.

Henggeler, S. W., Melton, G. B., Scherer, D. G., \& Hanley, J. H. (1997). Multisystemic therapy with violent and chronic juvenile offenders and their families: The role of treatment fidelity in successful dissemination. Journal of Consulting and Clinical Psychology, 65(5), 821-833.

Lehman, W., Greener, J., \& Simpson, D. (2002). Assessing organizational readiness for change. Journal of Substance Abuse Treatment, 22, 197-209.

Lyons, J. S. (2009). Communimetrics: A communication theory of measurement in human service settings. New York: Springer.

McGrew, J. H., Bond, G. R., Dientzen, L., \& Salyers, M. (1994). Measuring the fidelity of implementation of a mental health program model. Journal of Consulting and Clinical Psychology, 62(4), 670-678.

Metz, J. R., Blasé, K., \& Bowie, L. (2007). Implementing evidence based practices: Six "drivers" of success. In Research-to-results, child trends. Publication 2007-29. The Atlantic Philanthropies.

Miles, P., Bruns, E. J., Osher, T. W., Walker, J. S., \& the National Wraparound Initiative Advisory Group. (2006). The wraparound process user's guide: A handbook for families. Portland, OR: National Wraparound Initiative, Research and Training Center on Family Support and Children's Mental Health, Portland State University.

Prochaska, J. O., Norcross, J. C., \& DiClemente, C. C. (1994). Changing for good: A revolutionary six-stage program for overcoming bad habits and moving your life positively forward. New York: HarperCollins.

Rogers, E. M. (2003). Diffusion of innovations (5th ed.). New York: Free Press.

SPSS. (2010). PASW statistics: Version 18.0. Chicago, IL: SPSS, Inc.

Stephens, R. L., Holden, E. W., \& Hernandez, M. (2004). System-of-care practice review scores as predictors of behavioral symptomology and functional impairment. Journal of Child and Family Studies, 13(2), 179-191.

Suter, J., \& Bruns, E. (2009). Effectiveness of the wraparound process for children with emotional and behavioral disorders: A meta-analysis. Clinical Child Family Psychology Review, 12, 336-351.

Walker, J. S. (2008). Supporting wraparound implementation: Overview. In E. J. Bruns \& J. S. Walker (Eds.), The resource guide to wraparound. Portland, OR: National Wraparound Initiative, Research and Training Center for Family Support and Children's Mental Health.

Walker, J. S., Koroloff, N., \& Schutte, K. (2003). Implementing high-quality collaborative individualized servicel support planning: Necessary conditions. Portland, OR: Research and Training Center on Family Support and Children's Mental Health, Portland State University.

Walker, J. S. \& Sanders, B. (2010). The community supports for wraparound inventory: An assessment of the implementation context for wraparound. Journal of Child and Family Studies. doi:10.1007/s10826-010-9432-1.

Walton, B. A. (2006). Predictors of improvement for children served in developing systems of care. Doctoral dissertation, Indiana University. Proquest, 3242774.

This manuscript was published in the Journal of Child and Family Studies, 20, 736-746, in 2011. The original publication is available at www.springerlink.com. 